\title{
Extra-articular tibial deformity management in total knee replacement
}

\author{
Sanjeev Agarwal, Sarah Chol, Rakesh Kumar, Rhidian Morgan-Jonnes
}

From the University Hospital of Wales, Cardiff, UK

\begin{abstract}
Background : Presence of an extra-articular deformity in the femur or tibia poses a challenge to the surgeon undertaking knee replacement procedure. The conundrum is whether to correct the deformity beforehand, or accept the deformity and compensate for this through placement of the implant.

Material and Methods : This is a retro-spective study comprising six patients who had a knee replacement in the presence of an extra-articular deformity of the tibia treated at our centre. All six had the knee replacement without correction of deformity. The data evaluated included clinical outcome, mechanical axis correction, type of implant, and the use of any software / computer guidance.

The deformity was managed through planning of tibial resection without the need for pre-operative deformity correction.

Results : Mean age was 66.5 years. Mean coronal plane deformity in the tibia was 8.6 degrees. The hipknee-ankle improved from a mean 12.6 degrees to 4 degrees. Mean Oxford knee score improved from a mean of 19 to 33.6.

Conclusion: Planning the tibial resection on the basis of mechanical axis of tibia allows correction of alignment without the need for preoperative correction.

Correction of the deformity may not be needed if the maximum tibial resection is less than $15 \mathrm{~mm}$.
\end{abstract}

Keywords : Knee replacement ; knee arthroplasty ; tibia malunion ; tibia ; deformity.

No benefits or funds were received in support of this study. None of the authors have a conflict of interest.

\section{INTRODUCTION}

Restoration of mechanical alignment continues to be a fundamental tenet of knee replacement surgery. In the vast majority of patients, this can be achieved reliably with standard instrumentation.

Patients with extra-articular deformity (EAD) pose challenges in planning knee replacement. In the presence of significant deformity, the surgeon is faced with the dilemma of correction of deformity prior to replacement, or correction at the same time as replacement, or indeed leaving the deformity uncorrected and planning the replacement to compensate for the malalignment. What constitutes a significant deformity remains undefined as the distance and direction of the deformity from the knee joint act as confounding factors to the calculations. A deformity closer to the knee may have a greater impact on the functioning of the joint

Sanjeev Agarwal ${ }^{1}$, FRCS Orth Consultant Orthopaedic Surgeon

Sarah $\mathrm{Choi}^{1}, \mathrm{MBChB}$, , Surgical trainee

- Rakesh Kumar $^{2}$, FRCS Orth. Consultant Orthopaedic Surgeon,

- Rhidian Morgan-Jones', FRCS Orth, Consultant Orthopaedic Surgeon

${ }^{1}$ University Hospital of Wales, Cardiff, UK

${ }^{2}$ Prince Charles Hospital, Merthyr Tydfil, UK

Correspondence : Mr. Sanjeev Agarwal, University Hospital of Wales, Cardiff, UK.

Email : sagarwa125@gmail.com

- 2021, Acta Orthopædica Belgica. 
(1). The direction of deformity can be considered in the coronal plane, sagittal plane and the axial plane.

Computer navigation for knee replacement is a useful tool in such situations, although the number of knee replacements done using computer guidance remains low in the UK at $3 \%$. The navigation systems are configured to restore mechanical axis (2). In severe deformities, this may involve unconventional bone resection, which makes soft tissue balancing difficult to achieve with standard unconstrained implants (3). Despite the use of computer system, it is helpful for the surgeon to have a clear aim of the desired postoperative alignment.

We present an algorithm based on our experience and existing literature on planning knee replacement surgery in the presence of extraarticular coronal plane deformity of the tibia.

\section{MATERIAL AND METHODS}

We analysed 6 patients with coronal plane EAD of the tibia who underwent knee replacement at our centre in the last 10 years. Computer navigation system is in regular use in our centre for difficult primary knee replacements and patients with extraarticular deformity.

The degree of the coronal plane deformity of the tibia was measured on limb alignment radiographs from the hip to the ankle joints. The radiographs are obtained with a standard positioning protocol whereby the patella is facing anteriorly. The $\mathrm{x}$ ray beam is centred on the knee and is projected perpendicular to the knee joint. Patients stand barefoot with the feet parallel to each other in the 'attention position' as described by Paley (4).

Lateral radiograph of the tibia demonstrated any sagittal plane deformity. Rotational alignment was checked clinically.

The mechanical axis of the limb was assessed as described by Bellemans (5). The mechanical axis of femur was drawn from the centre of the femoral head to the midpoint of intercondylar line. The tibial mechanical axis was from the centre of the tibial spines to the midpoint of talar width. An angular deviation between the mechanical axis of femur and tibia was designated as varus or valgus mechanical axis of the limb. This angle is designated as the Hip Knee Ankle angle (HKA angle).

Preoperative and postoperative clinical score were recorded. The type of prosthesis, degree of constraint used, and any post-operative complications were recorded. A postoperative long leg alignment radiograph was obtained for all patients in the standing position to document the postoperative limb alignment.

This study is a retrospective analysis and was approved as a service evaluation project from the local institutional ethical committee.

\section{RESULTS}

The mean age of patients at the time of knee replacement was 66.5 years. All were men. The time interval between the tibial fracture and the knee replacement operation was 31 years. All patients were managed nonoperatively for the tibial fracture, and consequently, no patient had retained metalwork. One patient had an external fixator for tibial lengthening 8 years prior to the knee replacement.

The mean coronal plane deformity was 8.6 degrees (range 5 to 11 degrees). Four patients had deformities in the mid third, one in the distal third and one patient had a segmental fracture involving the mid third of tibia. In this patient, the angle between the proximal tibia and distal tibia was considered for angular measurements, ignoring the healed intermediary segment.

Three patients had varus deformity and three had valgus deformity. No rotational correction was required in any of these patients. None of the patients had a sagittal plane deformity greater than 5 degrees, and no sagittal correction was planned.

All patients had total knee replacement without correction of deformity. The knee replacement was carried out with computer navigation system in four patients and using a preoperative planning software (Orthoview, Hampshire, England) in the remaining two patients.

Adequate alignment of the limb and restoration of the mechanical axis was achieved in all six patients without the need for correction of tibial deformity. All patients had an unconstrained 
posterior stabilised prosthesis. No stems were used on the femoral or tibial side.

The preoperative mechanical axis (Hip Knee Ankle angle) was 12.6 degrees. Mean postoperative HKA angle was 4 degrees, giving a mean correction of 8.6 degrees. Mean preoperative Oxford knee score was 19 (11 to 27) and postoperative score was 33.6 (24 to 44). All six patients had improvement in score. No reoperations were needed in any patient and no significant postoperative complication was recorded. Mean follow up after the knee replacement procedure was 4.5 years (2-15 years).

\section{DISCUSSION}

With improvements in trauma management and widespread use of internal fixation, malunions of the femur and tibia are rarely seen in modern practice. Varus malunion can contribute to medial compartment arthritis of the knee (6).

Most of the patients with tibial malunions encountered in modern practice would have been managed decades previously, or had overriding associated problems that precluded achievement of anatomical alignment. EAD is defined as a deformity beyond the attachment of the collateral ligaments.

EAD management continues to cause consternation to the knee surgeon. Due to the relative rarity of patients with EAD in a surgeon's practice, the reported case series in literature have relatively few patients. Moreover, the severity of the deformity and its location in the tibia are further confounding factors, which obfuscate the decision making process. A suggested number is 15 degrees (7) deviation from the mechanical axis.

Our series has limited number and we have focussed on tibial deformities only. In our patients, the deformity was relatively mild (mean 8.6 degrees). In most developed healthcare system around the world, deformities greater than this would be addressed early to maintain optimum function and prevent problems in the long term.

Deschamps (8) reported a retrospective multicentre review of 36 tibial and further 4 more combined tibial and femoral mal-unions. In their series, 22 degrees varus of tibia and 15 degrees valgus of tibia was managed without prior corrective osteotomy. Good results with knee replacement without correction of deformity were demonstrated. Although the preoperative planning was not detailed, they describe a 'capsuloligamentous envelop' which is the safe zone for tibial and femoral resection. The exact boundaries of the envelop have not been defined.

While the degree of deformity has been considered, the anatomical site of the deformity is not considered in the pre-operative planning.

Mullaji (3) reported on 18 patients with tibial deformity managed with computer aided knee replacement surgery. Three patients had a concurrent proximal tibial corrective osteotomy stabilised with a stemmed tibial component. The degree of deformity in the tibia is not detailed in the article, as it has been grouped with patients with femoral deformity.

Details of preoperative planning are not available.

Another report (9) of 10 patients with proximal tibial deformity showed good results with intraoperative correction achieved with a proximal tibial osteotomy. None of the patients had diaphyseal or distal tibial deformities. No preoperative planning was done and the residual deformity after proximal tibial resection was addressed by the tibial osteotomy.

Catonnea (10) reported on 26 knee replacements in 25 patients where an extra-articular deformity was corrected through an osteotomy at the time of knee replacement. Limited information is available regarding planning. The osteotomy was stabilised through a stemmed tibial component.

Wang (11) described a method for planning knee replacement in the presence of EAD. Their study group included eight patients with tibial deformity and seven with femoral deformity. Wang's method has been documented in several papers $(12,13)$.

The Wang method for femoral deformities relies on the integrity of the collateral ligaments. For planning the femoral implant positioning, the femoral mechanical axis is drawn and a perpendicular to the axis is drawn at the level of the femoral condyle. The exact point on the condyle where the perpendicular has to be drawn was not precisely descried in the article, but the illustration suggests that this is drawn 
at the level of the intercondylar notch. The collateral attachment is 20 to $25 \mathrm{~mm}$ from the joint line and if the planned distal femoral resection compromises the femoral attachment of the collaterals, then an intra-articular correction would not be advisable. In that situation, the deformity should be corrected prior to knee replacement surgery.

On the tibial side, an entirely different approach has been adopted. The Wang method relies heavily on distal fragment alignment and the need for correction is dictated by the orientation of the distal fragment. The disadvantage with this approach is that relatively minor deformities, if further from the joint, can misleadingly dictate the need for correction.

The impact of minor distal tibial deformities on the mechanical axis of the tibia, and the function of the knee joint is small.

In our method, the mechanical axis of tibia is considered and the positioning of the tibial component is planned perpendicular to the mechanical axis of tibia, as it was in the femoral planning. The integrity of the collaterals determines the need for correction of deformity. A tibial resection of greater than $15 \mathrm{~mm}$ from medial or lateral aspect of tibia is an indication for corrective osteotomy prior to replacement surgery. The 15 $\mathrm{mm}$ resection is chosen for two reasons. First, the integrity of the collateral ligaments may be compromised with greater resection of proximal tibia. The intention is to keep the osteotomy within the 'envelope' of the collateral ligaments. Secondly, greater resection will require reconstruction with thicker polyethylene and more extensive soft tissue release on the opposite side, and this may adversely influence the function of the knee joint.

Our method is similar to that described by Sculco et. al. (14) in a review article. They recommend drawing the mechanical axis of tibia to assess the amount of tibial resection. The amount of resection that can be managed with intra-articular correction has not been defined in the article.

Our methodology is illustrated in the example. In Figure 1a, planning using the Wang method is illustrated. The line along the distal fragment passes beyond the margins of the proximal tibial articular surface and hence this deformity would require correction. However, in our method (Figure 1b), the perpendicular to the tibial axis is within $15 \mathrm{~mm}$ of the proximal tibial articular surface (Figure 2), and hence this deformity would be manageable using a standard knee replacement. Preoperative correction of deformity is not required.

Figure 3 is the postoperative appearance showing adequate correction, well balanced knee using a primary unconstrained implant and restoration of mechanical axis of the limb. The natural mechanical axis for this patient passes through the medial tibiofemoral compartment due to the constitutional varus.

As a result of this, we feel that relatively small coronal plane deformities in the lower third of the leg may lead the surgeon towards correction of deformity while these could easily and reliably be managed without correction.

Correction of deformity at the Centre of Rotation and Angulation (CORA) in the lower third of the
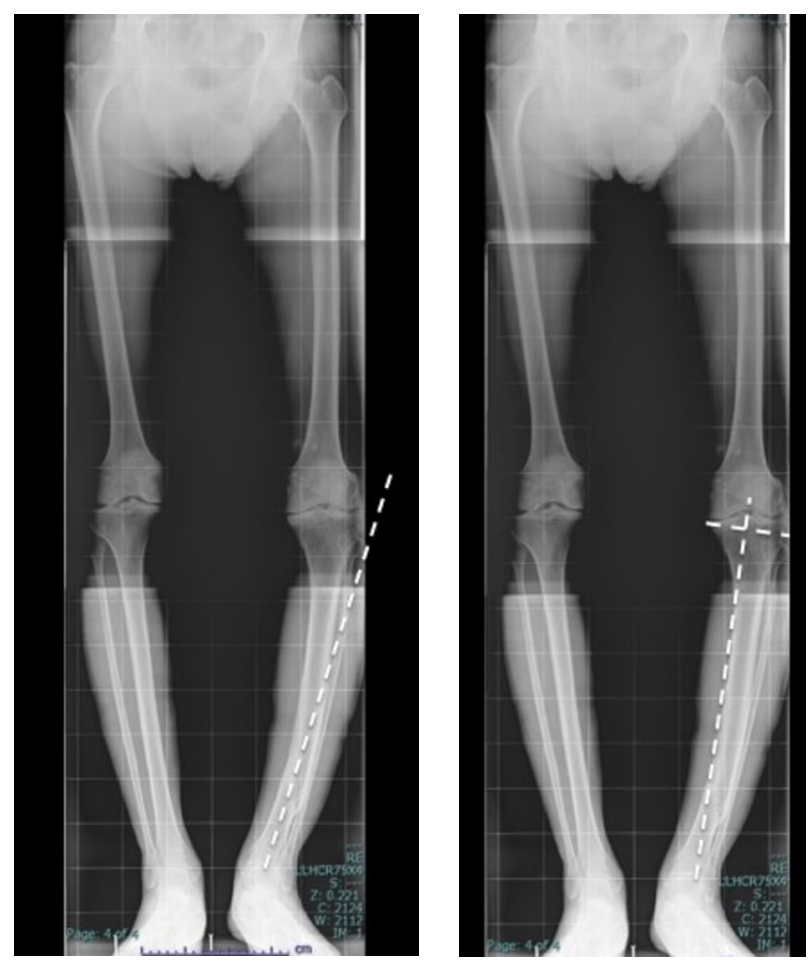

Figure 1. - a. Long leg alignment view showing a left sided distal tibial varusmalunion. Planning using the Wang method. $\boldsymbol{b}$. Long leg alignment view of the same patient showing planningusing our method. 


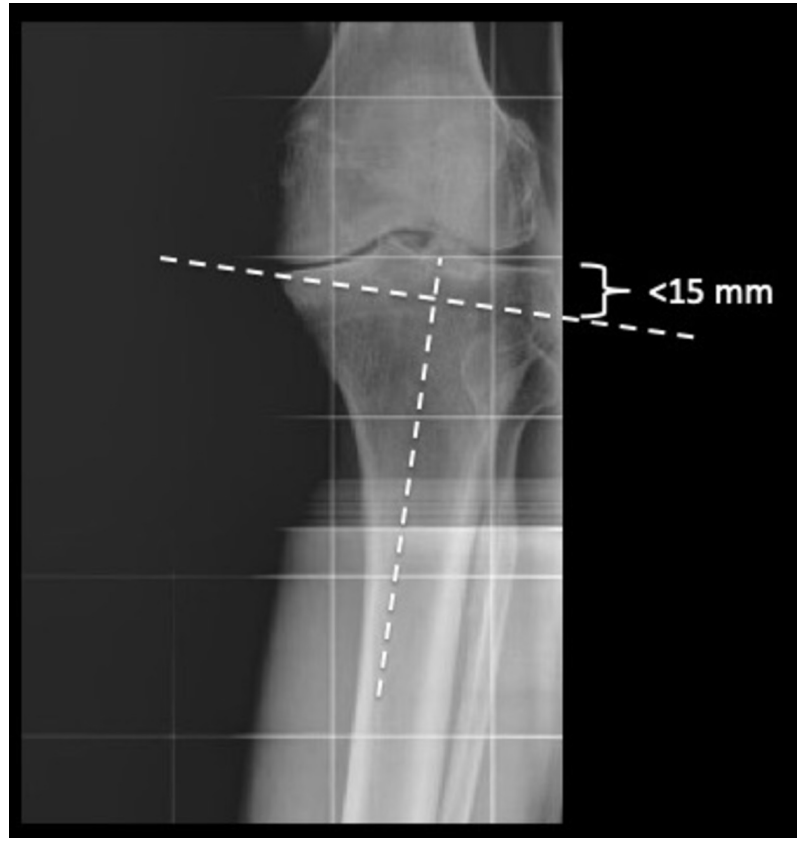

Figure 2. - The maximum bone resection of proximal tibia should be less than $15 \mathrm{~mm}$.

leg exposes the patient to an additional procedure, which carries a high risk of complications, delayed union and prolonged recovery.

Our study, as does most of the literature on this subject, suffers from limited number of patients. It is conceivable that the number of patients with significant deformity will reduce further as management of trauma improves. Due to the rarity of such cases, prospective studies or comparative studies are difficult to plan. Additionally, the most of the reported literature is before the days of widespread availability of computer navigation assisted surgery. Adequate planning for correction has not been described in literature.

We have employed computer navigation, and preoperative planning software to manage the deformities with the use of standard unconstrained implants.

It has to be borne in mind that the maximum deformity may not perpendicular to the plane of the radiographs. This method does not account for correction of recurvatum or procurvatum deformities, or rotational deformities. However, the impact of these on the long term survival of the knee implant remains unknown.

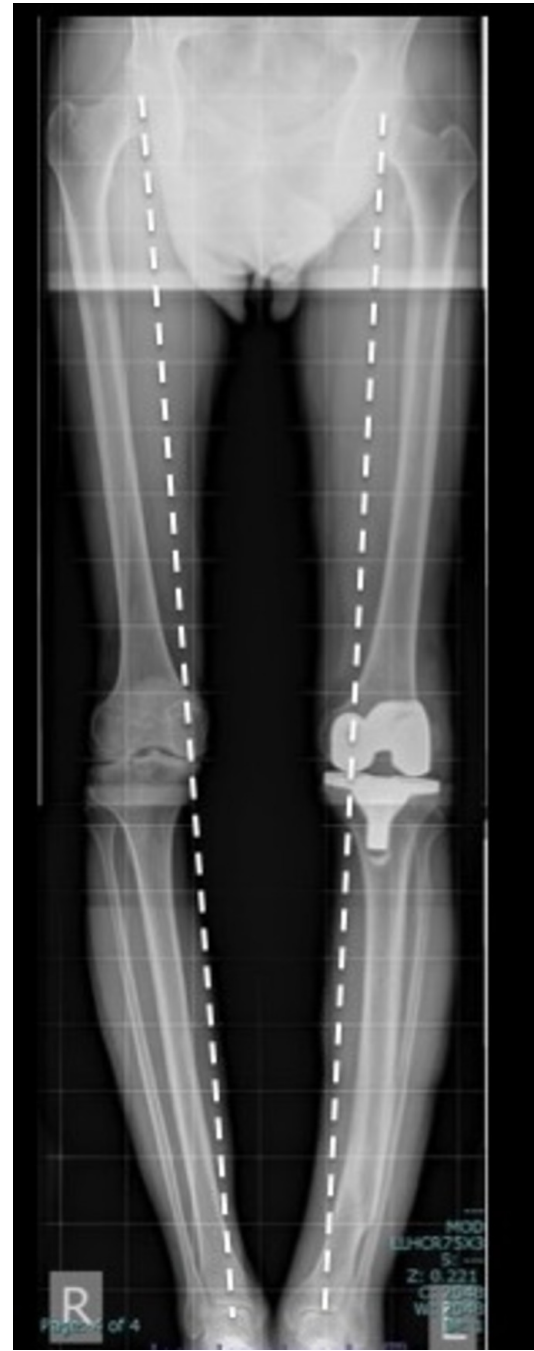

Figure 3. - Postoperative alignment view showing restoration of mechanical axis without need for corrective osteotomy.

This study will help provide guidance on planning and decision making for patients with extra-articular coronal plane tibial deformities.

\section{REFERENCES}

1. Wolf AM, Hungerford DS, Pepe CL. The effect of extra-articular varus and valgus deformity on total knee arthroplasty. Clin Orthop Relat Res 1991 ; 271 : 135- 51.

2. Catani F, Digennaro V, Ensini A, Leardini A, Giannini S. Navigation- assisted total knee arthroplasty in knees with osteoarthritis due to extra- articular deformity. Knee Surg Sports Traumatol Arthrosc 2012 ; 20 : 546-51. 
3. Mullaji A, Shetty GM. Computer-assisted total knee arthroplasty for arthritis with extra-articular deformity. $J$ Arthroplasty 2009 ; 24 : 1164-9.

4. Paley D. Principles of deformity correction. Heidelberg, Germany, Springer-Verlag : 2003 ; 1-60.

5. Bellemans J, Colyn W, Vandenneucker H, Victor J. Is neutral mechanical alignment normal for all patients? The concept of constitutional varus. Clin Orthop Rel Res 2012 ; 470(1) : 45-53.

6. Milner SA, Davis TRC, Muir KR, Greenwood DC, Doherty M. Long term outcome after tibial shaft fracture : is malunion important. J Bone Joint Surg Am 2002 ; 84(6) : 971-80.

7. Wang JW, Chen WS, Lin PC, Hsu CS, Wang CJ. Total knee replacement with intra-articular resection of bone after malunion of a femoral fracture : can sagittal angulation be corrected? J Bone Joint Surg [Br] 2010 ; 92B : 1392-6.

8. Deschamps G, Khiami F, Catonné Y, Chol C, Bussière C, Massin P. Total knee arthroplasty for osteoarthritis secondary to extra-articular mal-unions. Orthop Traumatol Surg Res 2010 ; 96 : 849-55.

9. Radke S, Radke J. Total knee arthroplasty in combination with a one- stage tibial osteotomy : a technique for correction of a gonarthrosis with a severe ( $>15$ degrees) tibial extra-articular deformity. J Arthroplasty 2002; 17 : 533-7.

10. Catonnéa Y, Sariali E, Khiamia F, Rouvillaind J-L, Wajsfisza A, Pascal- Moussellard H. Same-stage total knee arthroplasty and osteotomy for osteoarthritis with extraarticular deformity. Part I : Tibial osteotomy, prospective study of 26 cases. Orthopaedics \& Traumatology : Surgery \& Research 2019; 105 : 1047-54.

11. Wang JW, Wang CJ. Total knee arthroplasty for arthritis of the knee with extra-articular deformity. J Bone Joint Surg Am 2001 ; 84(10) : 1769-74.

12. Loures FB, Correia W, Reis JH, Pires E Albuquerque RS, de Paula Mozela A, de Souza EB, Maia PV, Barretto JM. Outcomes after knee arthroplasty in extra-articular deformity. Int Orthop 2019 ; 43(9) : 2065-2070.

13. Fan JC, Kwok KB, Hung YW. Total knee arthroplasty in a knee with triple deformity of femur-tibia-extensor mechanism. J Orthop Surg (Hong Kong) 2018 ; 26(1) : 2309499017718911.

14. Sculco PK, Cynthia A. Kahlenberg CA, Fragomen AT, Rozbruch SR. Management of extra-articular deformity in the setting of total knee arthroplasty. J Am Acad Orthop Surg $2019 ; 27(18)$ : e819-e830. 